151 NOX5 INDUCES VASCULAR DYSFUNCTION AND ARTERIAL REMODELLING INDEPENDENTLY OF BLOOD PRESSURE ELEVATION IN ANG II-INFUSED NOX5EXPRESSING MICE

${ }^{1}$ Augusto Montezano*, ${ }^{1}$ Adam Harvey, ${ }^{1}$ Francisco Rios, ${ }^{1}$ Wendy Beatie, ${ }^{1}$ Laura McPherson, ${ }^{1}$ Jacqueline Thomson, ${ }^{2}$ Chet E Holterman, ${ }^{2}$ Christopher Kennedy, ${ }^{1}$ Rhian M Touyz. ${ }^{1}$ University of Glasgow; ${ }^{2}$ University of Ottawa

10.1136/heartjnl-2017-311726.150

Nox5 is a unique $\mathrm{Ca}^{2+}$-sensitive Nox isoform that is expressed in human vascular smooth muscle cells (VSMC). Although Nox5 has been implicated in diabetic nephropathy, its role in vascular function and development of hypertension remain unclear. Nox 5 is not expressed in rodents, and accordingly we generated humanised Nox5 mice with Nox5 expressed in a VSMC-specific manner (Nox5SM22). Control (wild-type) and Nox5SM22 mice were infused with Ang II $(600 \mathrm{ng} / \mathrm{Kg} /$ day). Blood pressure (BP) was assessed by tail-cuff. Vascular function and structure of resistance arteries were measured by myography. Ang II increased BP in WT and Nox5SM22 mice with no significant differences. Arteries from Nox5SM22 mice exhibited reduced endothelium-dependent relaxation versus WT controls (\%ACh relaxation: $55.1 \pm 4$ vs ctl: $81.6 \pm 7 \%$ ). Fasudil (Rho kinase inhibitor)-induced relaxation was reduced in Nox5SM22 mice versus controls (\%Fas relaxation: $111.3 \pm 11$ vs ctl: $166.6 \pm 8 \%) \quad(\mathrm{p}<0.05)$. Ang II increased the maximal contraction to U46619 (thromboxane A2 mimetic) in WT $(115.8 \pm 2$ vs untreated: $101.4 \pm 2 \%)$ and Nox5SM22 (121.3 \pm 3 vs untreated: 99.1 \pm 2$) \quad(\mathrm{p}<0.05)$ and induced endothelial dysfunction in all groups. Fasudil-induced relaxation was impaired by Ang II in WT (102.7 \pm 6 vs untreated: $166.6 \pm 8 \%, \mathrm{p}<0.05)$ but not further impaired in Nox5SM22 mice (114.9 \pm 6 vs untreated: $111.3 \pm 11 \%)$. Ang II increased cross-sectional area (CSA) and lumen diameter) while in Nox5SM22 mice, Ang II increased wall thickness, wall-tolumen ratio, CSA and decreased lumen diameter, with associated increased vascular stiffness. Our findings indicate that in mice expressing human Nox5 in VSMCs, endothelium-dependent relaxation is impaired, fasudil-mediated vasodilation is attenuated and vessels undergo exaggerated hypertrophic inward remodelling with increased stiffness; processes that occur independently of BP elevation. These data suggest an important role for Nox5 in Ang II-induced vascular dysfunction and remodelling, but not in the development of hypertension. Moreover, we identify Rho kinase as a putative target for Nox5-induced vascular injury. We provide novel insights into Nox5 vascular biology and demonstrate that vascular Nox5 actions are dissociated from BP effects.

\section{THE ROLE OF ADAMTS-5 IN EXTRACELLULAR MATRIX REMODELLING OF THORACIC AORTIC ANEURYSMS}

${ }^{1}$ Marika Fava*, 'Javier Barallobre-Barreiro, ${ }^{1}$ Ferheen Baig, ${ }^{1}$ Ursula Mayr, ${ }^{1}$ Marc Lynch, ${ }^{1}$ Abhishek Joshi, ${ }^{1}$ Norman Catibog, ${ }^{1}$ Renata Gomes, ${ }^{1}$ Temo Barwari, ${ }^{2}$ Pouya Youssefi, ${ }^{2}$ Alessandro Viviano, 'Xiaoke Yin, ${ }^{2}$ Marjan Jahangiri, 'Manuel Mayr. ${ }^{1}$ King's College London; ${ }^{2}$ St. Georges Hospital, University of London

\subsection{6/heartjnl-2017-311726.151}

Introduction Thoracic aortic aneurysms (TAA) are common in patients with bicuspid aortic valve (BAV). ADAMTS-1 (a disintegrin and metalloproteinase with thrombospondin motifs) has recently been implicated in TAA formation (Oller et al, Nat Med, 2017). The contribution of other ADAMTS proteases to TAA is currently unknown.

Method Using proteomics, we compared the extracellular matrix (ECM) composition in the greater (i.e. the aneurysmprone area) and lesser curvatures of TAA in BAV patients. Our findings in patients were complemented by studies in ADAMTS-5 deficient mice.

Results In BAV patients with TAA, the large aggregating proteoglycan versican was the most differentially regulated ECM protein in the aneurysm-prone area. In mice, ADAMTS-5 is the main versican-degrading member of the ADAMTS family. Hence, a model of aortic dilatation by angiotensin II (AngII) infusion was adopted in mice lacking the catalytic domain of ADAMTS-5 (Adamts-5 ${ }^{\text {cd }}$ ). AngII treatment raised blood pressure in wild-type (WT) mice; this response was attenuated and associated with increased dilation of the ascending aorta in Adamts- $5^{\text {cd }}$ mice. Concomitantly, versican accumulation and reduced versican degradation products were observed in Adamts- $5^{\text {cd }}$ aortas compared to WT controls. The presence of other ADAMTS members, including ADAMTS-1, was not sufficient to maintain versican processing and prevent aortic dilation in Adamts- $5^{\mathrm{cd}}$ mice.

Conclusion Our results support the emerging role of ADAMTS proteases in TAA. ADAMTS-5 rather than ADAMTS- 1 is the key protease for versican regulation in murine aortas. Further studies are needed to define the ECM substrates of the different ADAMTS proteases and their contribution to TAA formation.

\section{METABOLIC DISORDER-INDUCED GLOBAL NOX2 ACTIVATION AND ENDOTHELIAL DYSFUNCTION IN AGEING}

${ }^{1}$ Li Geng*, ${ }^{2}$ Lampson M Fan, ${ }^{1}$ Jian-Mei Li. 'University of Reading; ${ }^{2}$ University of Oxford

\subsection{6/heartjil-2017-311726.152}

Ageing is an independent risk factor of cardiovascular diseases which involves oxidative stress derived from a Nox2-containing NADPH oxidase. However, the mechanism of endothelial Nox2 activation in normal ageing process remains unclear. In this study, we investigated the therapeutic potential of targeting Nox2 in improving global metabolism and endothelial function at old age by using age-matched wild-type and Nox2 knockout mice at 3-4 months (young); 11-12 months (middle aged) and 21-22 months (ageing). Compared to young mice, middle-aged and ageing wild-type mice had significantly higher blood pressure, hyperglycaemia, hyperinsulinaemia. These were accompanied by oxidative stress in multiple organs including the lung, the liver, the heart and vessels. The vessel motor function was examined in an organ bath using aortas isolated from these mice. Endothelium-dependent vessel relaxation to acetylcholine was significantly impaired in aortas of wild-type ageing mice, and this was accompanied by increased expressions of Nox2 and markers of inflammation, activation of MAPK and Akt and decreased insulin receptor expression and function. However, these aging-associated disorders in the aortas were significantly reduced by knocking out Nox 2 in mice. In response to high glucose plus high insulin challenge, coronary microvascular endothelial cells isolated from wild-type mice displayed significantly increased Nox2 expression, oxidative stress and cell senescence, e.g. increase p53 expression 